\title{
Extramedullary Haematopoiesis Causing Spinal Cord Compression in a Thalassemia Intermedia Patient
}

\author{
Wahab $S^{1}$, Khan $R^{2}{ }^{2}$, Ahmad $K^{3}$, Wahab $A^{4}, \operatorname{Ahmad} I^{1}$ \\ ${ }^{1}$ Department of Radiodiagnosis,JNMCH, AMU Aligarh, ${ }^{2}$ Department of Surgery , JNMCH \\ ,AMU Aligarh, ${ }^{3}$ Department of Radio diagnosis , BPKIHS, Dharan, Nepal, ${ }^{4}$ Department of \\ Medicine ,JNMCH , AMU Aligarh, India
}

\begin{abstract}
The thalassemias are a heterogenous group of inherited disorders of hemoglobin $(\mathrm{Hb})$ synthesis characterized by a lack of or decreased /defective synthesis of globin chains. The name Mediterranean anemia, which Whipple introduced, is misleading because the condition can be found in any part of the world. In $\alpha$-thalassemia, $\alpha$-globin chain synthesis is reduced, whereas in $\beta$-thalassemia, $\beta$-globin chain synthesis is defective. Their clinical severity varies widely, ranging from asymptomatic forms to severe or even fatal entities. Like most anemias, erythropoietin production and erythropoiesis is increased in thalassemia causing erythroid marrow hyperplasia as well as extramedullary haematopoiesis which is a common compensatory mechanism for chronic anemia found in patients with hemoglobinopathies such as thalassemia, sickle cell anemia, and hereditary spherocytosis. These patients are usually asymptomatic. Extramedullary hematopoiesis (EMH) usually manifests in the liver, spleen, kidneys and as paravertebral masses. Extramedullary hematopoietic tissue occurring within the spinal canal and causing cord compression is an unusual but well-described entity. Total surgical excision is usually not feasible because of the diffuse spread of extramedullary hematopoietic tissue and the possibility of recurrence, but acute neurological impairment does require emergency surgery. Extramedullary hematopoiesis is radiosensitive so radiation therapy is widely accepted as a very effective mode of therapy. Repeated blood transfusion and cytostatic agents are also recommended for treatment. We report a case of a 35-year-old man with underlying beta thalassemia intermedia who developed pain in lower back and progressive paresthesia and weakness of both lower limbs due to multifocal extramedullary epidural hematopoietic tissue occurring within the spinal canal with compression over the cord.
\end{abstract}

Keywords: Extramedullary hematopoiesis, MRI, Spinal cord compression, Thalassemia

\section{Case Report}

A 35-year-old man with underlying

Corrospondence to: Dr. Rizwan Ahmad intermediate type beta-thalassemia presented with pain in lower back and progressive

Khan, Kashana e Wahab, Iqra Colony, New

Sir Syed Nagar, Aligarh- 202002.

Email: drrizwanahmadkhan@yahoo.co.in 


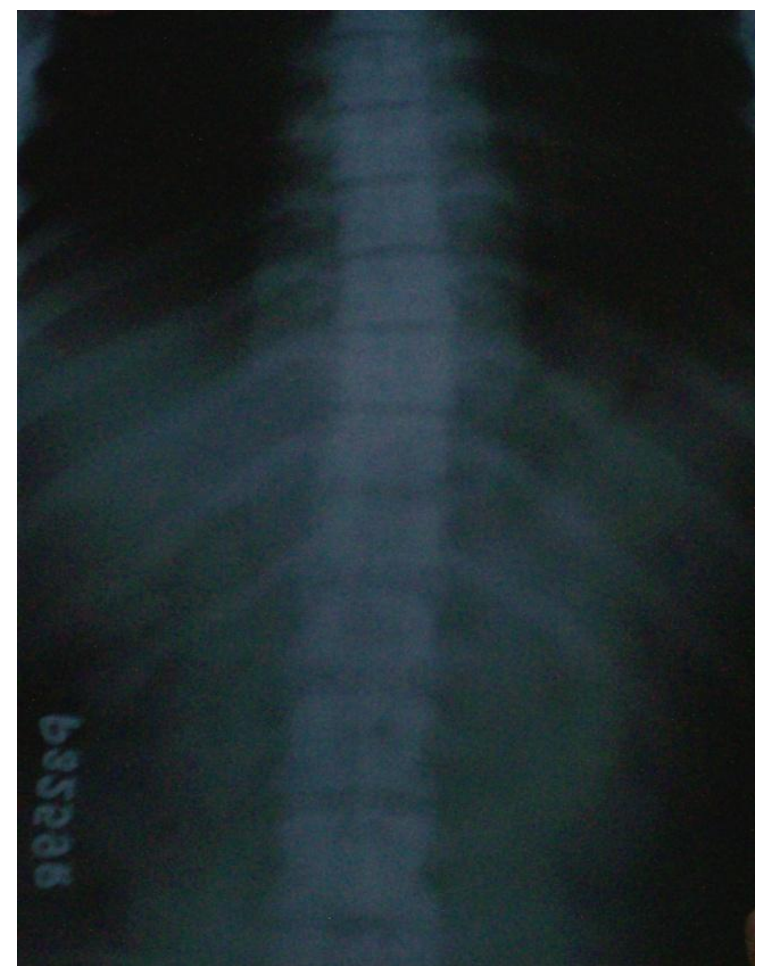

Fig 1: Radiograph of the dorsolumbar spine showing bilateral paravertebral softtissue masses with coarse trabeculae of vertebrae.

paresthesia and weakness of both lower limbs. He denied any history of trauma. $\mathrm{He}$ was not under any regular treatment for thalassemia except for one episode of blood transfusion six years back. Except for persistent anemia he denied any specific symptoms. On examination, his blood pressure was $110 / 76 \mathrm{mmHg}$, heart rate was 110 beats/min, and respiratory rate was 20 breaths/min. Cardiac examination revealed sinus tachycardia. Respiratory examination was normal. Hepatomegaly and splenomegaly was found on abdominal palpation. Nervous system examination revealed decreased power in both lower limbs 3/5 in right and 4/5 in left lower limb. His baseline hemoglobin was $5.5 \mathrm{~g} / \mathrm{dl}$. Haemogram examination revealed a white blood cell count of 70,000 cells $/ \mathrm{ml}$, and hematocrit was $32 \%$. Hemoglobin typing study showed Hb F -82.5\%; Hb A2 - 2.3\% and $\mathrm{Hb} \mathrm{A}-15.2 \%$. Liver function tests revealed total bilirubin $-2.4 \mathrm{mg} / \mathrm{dl}$, direct bilirubin $-0.4 \mathrm{mg} / \mathrm{dl}$, AST 47U/L, ALT 58U/L and alkaline phosphatase 93U/L.

Radiographs of the dorsolumbar spine showed bilateral paravertebral soft-tissue masses with generalized increased density and coarse trabeculae of vertebrae (Fig 1). X -ray skull was almost normal, however that of the hands revealed coarse trabeculation (Fig 2). Ultrasound abdomen revealed hepatomegaly and splenomegaly with large echogenic foci in spleen and a lobulated hypoechoic mass in presacral space which was indenting the urinary bladder in its lower part.

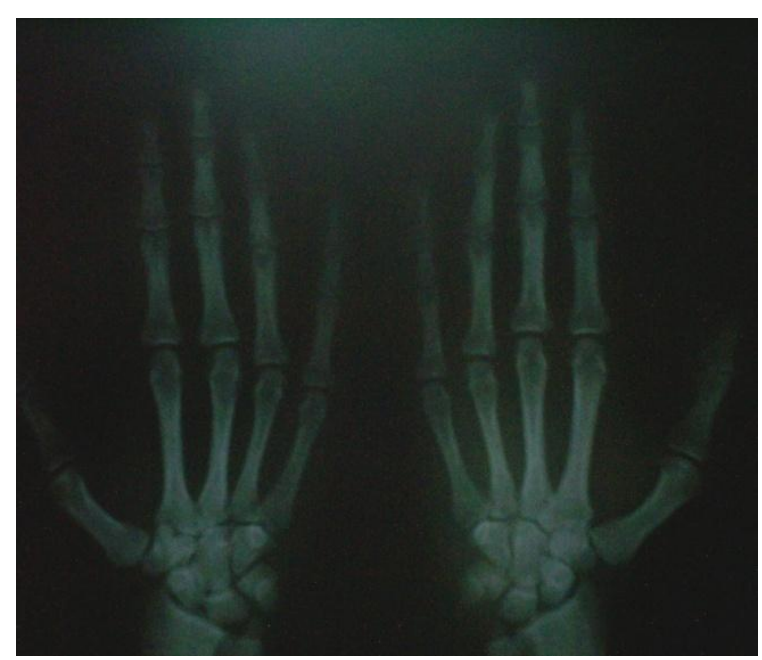

Fig 2: Radiograph of both hands showing coarse trabeculation.

MR Imaging of whole spine was performed which revealed multiple lobulated bilateral paravertebral, prevertebral and presacral masses isointense on both $\mathrm{T} 1$ and $\mathrm{T} 2$ weighted sequences (Fig 3,4 \& 5) with mild enhancement after IV administration of gadolinium (Fig 6). Epidural extension of these masses into spinal canal as well as other discrete epidural deposits of similar appearing masses were seen in the region of dorsal spine and sacrum.The lesions in 


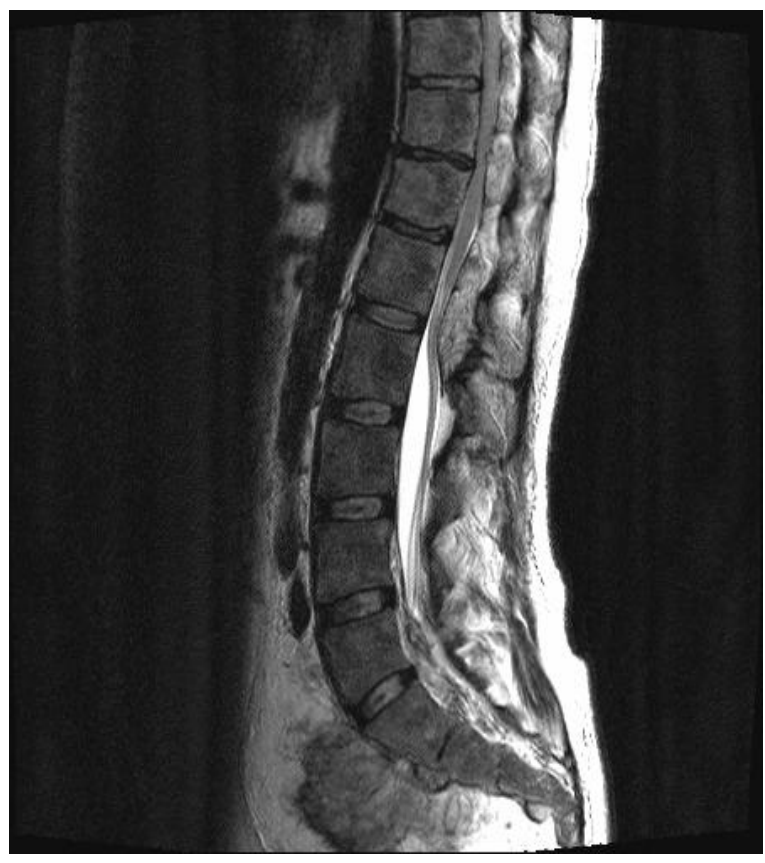

Fig 3: T2 weighted image showing isointense prevertebral and presacral masses with discrete epidural deposits causing compression over the spinal cord. Veretebral bodies also show altered signal intensity.

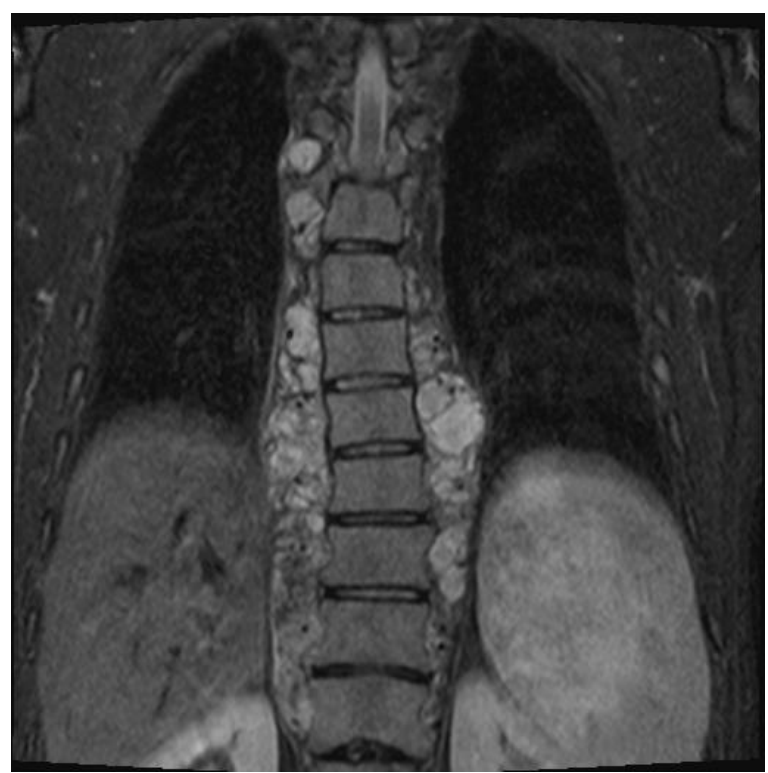

Fig 4: T2 weighted coronal image showing multiple foci of paravertebral masses and similar appearing focus in the enlarged spleen.

spleen showed similar signal intensity and enhancement pattern. All the vertebral

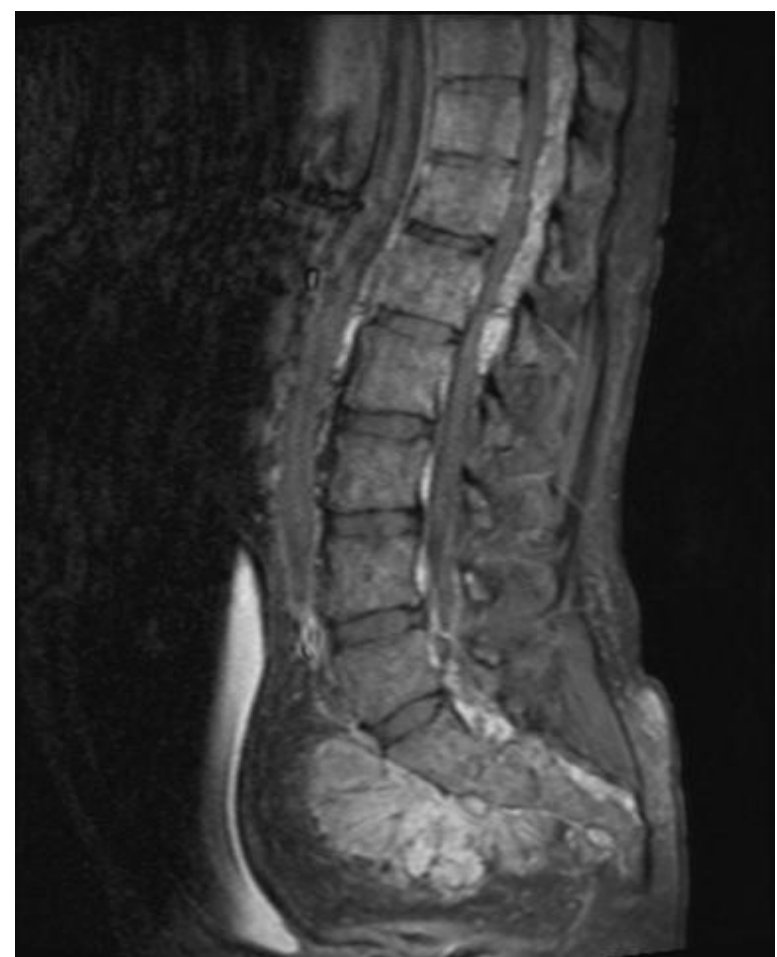

Fig 5: T2 weighted sagittal image showing multiple foci of prevertebral masses.

bodies had heterogenous low to intermediate signal intensity as a result of replacement of fatty marrow by haematopoietic marrow. The diagnosis of extramedullary haematopoiesis was made in view of the history of thalassemia and paravertebral, presacral and epidural soft tissue masses in the spinal canal consistent with imaging features of extramedullary hematopoietic tissue on MRI. Biopsy was avoided in view of the possibility of bleed.

At the time of writing this case report patient had undergone two blood transfusions and was advised radiation therapy.

\section{Discussion}

Extramedullary haematopoiesis usually develops as a compensatory response in patients of chronic anemia. It can occur in many disorders including hemolytic anemia, 
myelofibrosis, and other hemoglobinopathies but is particularly common in thalassemia intermedia patients who usually reach adult life without the need for frequent blood transfusion as their erythropoiesis is not suppressed. ${ }^{1}$ Patients with frequent blood transfusions and in whom haemoglobin level is maintained above $7 \mathrm{gm} / \mathrm{dl}$ rarely manifest extramedullary haematopoietic masses. ${ }^{1,2}$ Spinal cord compression due to EMH in thalassemia was first reported by Gatto in $1954 .^{3}$ This complication has been mainly observed in the thoracic segment of the spinal cord. ${ }^{4}$ Most cases present with neurological impairment as paraparesis, sensory impairment and occasionally sphincter disturbances. Complete paraparesis due to spinal cord compression by extramedullary hematopoietic tissue is very rare to occur in thalassemia and occurs more frequently in sickle cell anemia and polycythemia rubra vera. ${ }^{5}$ MRI appears to be the modality of choice for diagnosing and delineating the extent of the spinal cord compression by the haematopoietic masses. MRI usually shows isointense mass on both T1 and T2-weighted images with variable mild to intermediate contrast enhancement. The most important differential diagnosis is epidural lymphoma deposits. The vertebral bodies show low to intermediate signal intensity because of displacement of fatty marrow by haematopoietic marrow. ${ }^{6}$ Nuclear isotopic scans can show every ectopic foci of haematopoietic tissue ${ }^{7}$, but the gold standard remains MRI for complete evaluation of spinal cord compression. Most authors do not favor a tissue biopsy in this situation because of the risk of hemorrhage. ${ }^{4}$ History and clinical examination along with imaging features clinch the diagnosis as the patient would have a predisposing haematological condition. $^{8}$
The management of such patients remains controversial and consists of medical means (steroids, hydroxyurea, blood transfusions), surgical decompression and radiotherapy or their various combinations. Most cases of paraplegia due to extramedullary hematopoiesis have been treated with surgical decompression with or without radiation therapy. ${ }^{5}$ Hemopoietic tissue is extremely sensitive to radiation and low doses cause rapid shrinkage. A standard recommendation regarding the radiation dose is not available but a dose of 20Gy delivered over 10 fractions seems to be effective. ${ }^{9}$ Excellent results have been obtained in a few days with radiotherapy alone. Recurrence may occur but these cases can be treated with further radiation. Corticosteroids and blood transfusions are also used in treatment of these patients with the rationale of down regulating the hematopoiesis. However, improvement is usually incomplete and short lived. $^{10}$ Cytostatic agents such as hydroxyurea have also been used in extramedullary hematopoiesis with good results. ${ }^{11}$

In view of the excellent results obtained promptly with radiotherapy our patient was advised to undergo radiation therapy after two episodes of blood transfusion.

\section{Conclusion}

To conclude extramedullary hematopoiesis should be considered as a differential diagnosis in patients suffering from hematologic disorders presenting with back pain and spinal cord symptoms. MRI appears to be the modality of choice in complete evaluation and follow up of such patients. Early diagnosis and treatment with radiotherapy and /or surgery and blood 
transfusion is essential to prevent irreversible damage to the spinal cord.

\section{References}

1. Chute DJ, Fowler DR: Fatal hemothorax due to rupture of an intrathoracic extramedullary hematopoietic nodule .Am J Forensic Med Patol.2004; 25(1) 74-77.

2. Pornsuriyasak P, Suwatanapongched T, Wangsuppasawad $\mathrm{N}$ et al: Massive Hemothorax in a Beta -Thalassemic patient due to spontaneous rupture of extramedullary hematopoietic masses: Diagnosis and Successful Treatment .Respiratory Care 2006:51(3):272-6.

3. Gatto I, Terrana V, Biondi L. Compression sul spinale da proliferazione di midollo osseo nella spazio epidurale in soggetto affetto da malattia di Colley splenectomizzato. Hematologica 1954; 38:61-75.

4. Kaufmann T, Coleman M, Giardina P, Nisce LZ. The role of radiation therapy in the management of hematopoietic neurologic complications in thalassemia. Acta Haematol. 1991; 85:156-9.

5. Salehi SA, Koski T, Ondra SL. Spinal cord compression in beta-thalassemia: case report and review of literature. Spinal Cord 2004; 42:117-123.

6. Chourmouzi D, Pistevou-Gompaki K, Plataniotis G, Skaragas G, Papadopoulos L, Drevelegas A. MRI findings of extramedullary haemopoiesis. Eur Radiol. 2001; 11(9):1803-6.
7. Heffez DS, Sawaya R, Udvarhelyi GB, Mann R. Spinal epidural extramedullary hematopoisesis with cord compression in a patient with refractory sideroblastic anemia. J Neurosurg 1982;57: 399-406.

8. Alorainy IA, Al-Asmi AR, Del Carpio R. MRI features of epidural extramedullary hematopoiesis. Eur J Radiol 2000; 35:811.

9. Talapatra K, Nemade B, Siddha M, Muckaden MA, Laskar S. Extramedullary haematopoiesis causing spinal cord compression: A rare presentation with excellent outcome. Ann Indian Acad Neurol 2007;10:115-7.

10. Coskun E, Keskin A, Suzer T, Sermez Y, Kildaci T, Tahta K. Spinal cord compression secondary to extramedullary hematopoiesis in thalassemia intermedia. Eur Spine J 1998; 7:501-504.

11. Konstantopoulos K, Vagiopoulos G, Kantouni R, et al. A case of spinal cord compression by extramedullary haematopoiesis in a thalassemic patient: a putative role for hydroxyurea Haematologica 1992:77: 352-354. 